\title{
pH-Thermosensitive Oral Hydrogels Containing Phenobarbital for a Potiential Pediatric Use
}

\author{
Alphonse Rodrigue Djiboune ${ }^{1,2, *}$, Nicolas Anton ${ }^{2}$, Sidy Mouhamed Dieng ${ }^{1,2,4}$, Papa Mady Sy ${ }^{1,2}$, \\ Louis Augustin Diaga Diouf ${ }^{1}$, Gora Mbaye ${ }^{1}$, Mamadou Soumboundou ${ }^{3}$, Nadia Messaddeq ${ }^{6}$, \\ Said Ennahar ${ }^{5}$, Thierry François Vandamme ${ }^{2}$, Mounibe Diarra ${ }^{1, *}$ \\ ${ }^{1}$ Physical Pharmaceutic Laboratory, Faculty of Medicine, Pharmacy and Odontology, Cheikh Anta Diop University of Dakar, Dakar, Senegal \\ ${ }^{2}$ National Center for Scientific Research, Bioactive Molecule Design and Application Laboratory, Joint Research Unit 7199, University of \\ Strasbourg, Strasbourg, France \\ ${ }^{3}$ Biophysics Laboratory, Healthy UFR of Thies' University of Thies, Thies, Senegal \\ ${ }^{4}$ Galenic and Industrial Pharmacy Laboratory, Healthy UFR of Thies' University of Thies, Thies, Senegal \\ ${ }^{5}$ Multidisciplinary Institute Hubert Curien, Multidisciplinary Institute Hubert Curien-Department of Analytical Sciences, National Center for \\ Scientific Research, Joint Research Unit 7178, University of Strasbourg, Strasbourg, France \\ ${ }^{6}$ Institute of Genetics and Molecular and Cellular Biology, National Institute of Health and Medical Research U1258, National Center for \\ Scientific Research, Joint Research Unit 71104, University of Strasbourg, Strasbourg, France
}

Email address:

alphonserodrigue.djiboune@ucad.edu.sn (A. R. Djiboune), nicolas.anton@pharma.u-strasbg.fr (N. Anton), sidym.dieng@univ-thies.sn (S. M. Dieng), papamady.sy@ucad.edu.sn (P. M. Sy), louisaugustin.diouf@ucad.edu.sn (L. A. D. Diouf), Gora.mbaye@ucad.edu.sn (G. Mbaye), mamadou.soumboundou@univ-thies.sn (M. Soumbundou), nadiame@igbmc.fr (N. Masseddeq), ennahar@unistra.fr(S. Ennahar), vandamme@unistra.fr (T. F. Vandamme), mounibe.diarra@ucad.edu.sn (M. Diarra)

${ }^{*}$ Corresponding author

\section{To cite this article:}

Alphonse Rodrigue Djiboune, Nicolas Anton, Sidy Mouhamed Dieng, Papa Mady Sy, Louis Augustin Diaga Diouf, Gora Mbaye, Mamadou Soumboundou, Nadia Messaddeq, Said Ennahar, Thierry François Vandamme, Mounibe Diarra. pH-Thermosensitive Oral Hydrogels Containing Phenobarbital for a Potiential Pediatric Use. European Journal of Biophysics. Vol. 8, No. 2, 2020, pp. 43-51. doi: 10.11648/j.ejb.20200802.15

Received: September 22, 2020; Accepted: October 10, 2020; Published: October 16, 2020

\begin{abstract}
The purpose of this study was to develop a pH-thermosensitive oral chitosan-based hydrogels, able to release phenobarbital in the small intestine of the newborn. Phenobarbital is an active drug used in neonatal treatment of epilepsy. $\mathrm{pH}$ thermosensitive hydrogels will improve its bioavailability and therapeutic efficiency with less side effects. This study allowed us to understand the free energy variation at the interface between chitosan chains themselves and the surrounding available molecules with its interactions behavour. Indeed, inverted tube method was used to prepare hydrogels containing 2.45 and $2.55 \%$ of chitosan, eudragitE100 and phenobarbital at $37+/-1{ }^{\circ} \mathrm{C}$ via sol-gel transition. The characterization of their morphology was done by using XL SIRION 200 FEG SEM. In addition, conductivity, refractive index and density's values were determinated. The phenobarbital release mechanism from hydrogels at different $\mathrm{pH}$ values, simulating the gastrointestinal tract of the newborn was also studed. UV / visible spectrophotometer SHIMADZU from the UV-2400PC series was used to determine the phenobarbital released amount as a function of time. The results showed that sol-gel transition time decreases when the chitosan concentration increases. In addition, it showed that the hydrogels structure was heterogeneous and the phenobarbital released amount were more important at $\mathrm{pH}$ simulating the small intestine at $2.45 \%$ of chitosan, final solution's $\mathrm{pH} 6.85$ and with the presence of eudragitE100. These results were confirmed by conductivity's values. The sols and hydrogels had a comparable refractive index and density's values. The Korsmeyer-Peppas model was used to fit the phenobarbital release profiles. In short, the hydrogels formulated lend themselves to a phenobarbital pulsatile release usable in the newborn. The phenobarbital release profiles fitting makes possible to predict the phenobarbital amounts, which will be released at the action sites according to the need.
\end{abstract}

Keywords: $\mathrm{pH}$-Thermosensitive, Oral Hydrogels, Phenobarbital, Pediatrics, Pulsatile Release 


\section{Introduction}

Epilepsy is the first pediatrics neurological disease [1-4] and is a real public health problem. Phenobarbital is widely used for his care in the world [1, 5]. In sub-Saharan Africa, the oral form containing phenobarbital is a tablet and is unsuitable for newborns [6, 7]. In addition, with this form, phenobarbital is released in their stomach while the $\mathrm{pH}$ of the latter is neutral or basic [8-10]. This leads to an ionization of the molecule and a decrease of its bioavailability which can cause therapeutic failures [9]. For this purpose, the development and availability of new formulations adapted to age, size, physiological state and therapeutic requirements in accordance with the regulations of pediatric investigation plan (PIP) [7], and objectives of the pharmaceutical industry for neurological pathologies in pediatrics (epilepsy) [11], prove to be essential. It is in this context that we thought of developing a $\mathrm{pH}$-thermosensitive oral hydrogels containing phenobarbital, wich the main excipients are: chitosan, EudragitE100 and sodium bicarbonate. The choice of theses excipients was based on the fact that they are biocompatible, biodegradable and non-toxic $[12,13]$. As for hydrogels, they were used as vectors thanks to their controlled and targeted release properties of active drugs [12]. The purpose of this study was to formulate and characterize these hydrogels for a controlled and targeted oral administration of phenobarbital in the small intestine of the newborn. These hydrogels will improve the control of neonatal epileptic crises and his quality life with less side effects.

\section{Materials and Methods}

\subsection{Materials}

Phenobarbital base, chitosan with low molecular weight and more than $75 \%$ deacetylation degree (DAD), sodium bicarbonate and $99.8 \%$ acetic acid, purchased from Sigma Aldrich laboratories and eudragitE100, purchased from Evonik Röhm GmbH Pharma Polymers (Darmstadt, Germany) were used. All these excipients were used in powder form except acetic acid wich was used in liquid form. We had also used distilled water as solvent produced by a millipore system from Fisher Scientific Limited, Nepean ON, Canada. A water bath and a $\mathrm{pH}$ meter $781 \mathrm{pH} /$ ion meter (Metrohm) fitted with a temperature probe were used for the hydrogels preparation. For physicochemical characterization, konduktometer CG 854 (Schott Gerate), refractometer ATAGO (SPR-T2), a precision (0.1-1mg) Balance model bc (orma), scanning electron microscopy (SEM) type XL SIRION 200 FEG, Netherlands and UV / visible spectrophotometry SHIMADZU from the UV2400PC series were used.

\subsection{Methods}

\subsubsection{Hydrogels Preparations}

We prepared two batches of hydrogels: batch 1 and batch 2 . The first batch had included the formulations $F_{1}, F_{2}$, and $F_{3}$ while the second included the formulations $\mathrm{F}_{1}{ }_{1}, \mathrm{~F}_{2}{ }_{2}$, and $\mathrm{F}_{3}{ }_{3}$. Their composition and final solution's $\mathrm{pH}$ are indicated in table 1 .

1) For batch 1 (Chitosan/Sodium Bicarbonate-based hydrogels), we prepared a dilute acetic acid solution (2\% acetic acid) containing chitosan and phenobarbital. At the same time, we prepared a sodium bicarbonate aqueous solution at $1.12 \mathrm{M}$. The two solutions were first kept cool at a temperature between 1 and $2^{\circ} \mathrm{C}$ for $30 \mathrm{mn}$, then we added drop by drop the sodium bicarbonate aqueous solution in chitosan solution until a final solution's $\mathrm{pH}$ close to neutrality, is obtained. The final solution, obtained after mixing of the two solutions, was called sol. $5 \mathrm{ml}$ of this sol were transferred to glass tubes and then we put it in a water bath at $37+/-1^{\circ} \mathrm{C}$, to observe the sol-gel transition phenomenon every minute.

2) For batch 2 (Chitosan / EudragitE100 / Sodium Bicarbonate-based hydrogels), the preparation protocol was the same as the previous one. Except that, here the dilute acetic acid solution contained apart from chitosan and phenobarbital, eudragit E100.

\subsubsection{Physicochemical Characterization of Formulations}

\section{i. Visual and Organoleptic Aspect}

It consisted in observing with the naked eye the appearance of the formulated hydrogels and in smelling their odor.

\section{ii. Conductivity, Refractive Index and Density}

we determined the measurement of conductivity, refractive index and density of sols and hydrogels. The results of these measurements are mentioned in the table 2 .

\section{iii. Scanning Electron Microscopy}

A few milligrams of hydrogels had been collected and then frozen in nitrogen liquid and lyophilized for 48 hours. These dry samples had been placed on heels with carbon adhesive tabs, coated with gold and palladium in the spray coating (BAL-TEC SCD005), then examined using an XL SIRION 200 FEG SEM, Pays BAYS.

\section{iv. In vitro Release Study}

For this in vitro release study, $10 \mathrm{mg}$ of formulated hydrogels were placed in boro 3.3 type circular and transparent glass cubes with a volume equal to $125.6 \mathrm{~cm}^{3}$. These cubes were placed at the bottom of three beakers containing $500 \mathrm{ml}$ of dissolution medium (acetate buffers) at $\mathrm{pH}$ equal to 5 simulating the small intestinal liquid of newborns (prematures and term newborns) and (phosphatecitrate buffers) at $\mathrm{pH}$ equal to 7 for the gastric liquid of term newborns and at $\mathrm{pH}$ equal to 8 for the gastric liquid of premature $[8,10]$. These dissolution medium were kept stirring at $150 \mathrm{rpm}$ per minute at $37+/-1^{\circ} \mathrm{C}$. Regarding this in vitro release study, every 60 minutes, $4 \mathrm{ml}$ were taken from the dissolution medium and then replaced with fresh medium at each sampling point. The solutions collected were filtered using Nylon acrodisc 13 filters, which the diameter pore is equal to 0.45 micrometers, before any phenobarbital 
determination by UV / visible spectrophotometry at $215 \mathrm{~nm}$ for acetate buffer at $\mathrm{pH}$ equal to 5 , at $202 \mathrm{~nm}$ for phosphatecitrate buffer at $\mathrm{pH}$ equal to 7 and $204 \mathrm{~nm}$ at $\mathrm{pH}$ equal to 8 . For the dissolution medium simulating the intestinal liquids of the newborns (prematures and term newborns), the phenobarbital release study was carried out for $180 \mathrm{mn}$. Regarding the term newborns, the phenobarbital release study in the dissolution medium simulating his stomach, was carried out for $180 \mathrm{mn}$. On the other hand, for that of the premature, it was realized during $240 \mathrm{mn}$. The wavelengths were obtained by doing three spectral scans for different buffer containing phenobarbital. The cumulative percentage of phenobarbital was calculated from the phenobarbital amount initially present in the hydrogels compared to that measured in the release medium using the calibration curves equations (Equation 1, 2, and 3). The experiment was repeated three times.

$$
\begin{aligned}
& y=37.2 x+0.0155 ; R 2=0.9993(p H \text { equal to } 5) \\
& y=84.734 x+0.0054 ; R 2=0.9993(p H \text { equal to }) \\
& y=100.34 x+0.0408 ; R 2=0.9992(p H \text { equal to } 8)
\end{aligned}
$$

\subsubsection{Statistical Analysis}

Statistical significance $(\mathrm{P}<0.05)$ about the presence of eudragitE100, the variation of chitosan concentration and the final solution's $\mathrm{pH}$ between test intra and inter groups was determined by one-way analysis of variance (ANOVA) with

\begin{tabular}{|c|c|c|c|c|c|c|}
\hline Formulations & Chitosan (\%) & Eudragit E100 (\%) & Phenobarbital mg/ml & Sodium Bicarbonate (M) & Distilled water (g) & Final solution's pH \\
\hline $\mathrm{F}_{1}$ & 2.45 & - & 0.0293 & 1.12 & 45 & 6.85 \\
\hline$F_{1}^{\prime}$ & 2.45 & 0.0245 & 0.0293 & 1.12 & 45 & 6.85 \\
\hline $\mathrm{F}_{2}$ & 2.55 & - & 0.0293 & 1.12 & 45 & 6.85 \\
\hline $\mathrm{F}_{2}^{\prime}$ & 2.55 & 0.0255 & 0.0293 & 1.12 & 45 & 6.85 \\
\hline $\mathrm{F}_{3}$ & 2.45 & - & 0.0293 & 1.12 & 45 & 6.8 \\
\hline $\mathrm{F}_{3}$ & 2.45 & 0.0245 & 0.0293 & 1.12 & 45 & 6.8 \\
\hline
\end{tabular}
excel software.

Table 1. Formulations composition and final solution's $p H$.

\begin{tabular}{|c|c|c|c|c|c|c|c|c|c|c|c|c|}
\hline Formulations & $\begin{array}{l}\text { Conductivity } \\
\text { of sols }(\mathrm{S} / \mathrm{cm})\end{array}$ & SD & $\begin{array}{l}\text { Conductivity } \\
\text { of gels }(\mathrm{S} / \mathrm{cm})\end{array}$ & SD & $\begin{array}{l}\text { Refractive } \\
\text { index of sols }\end{array}$ & SD & $\begin{array}{l}\text { Refractive index } \\
\text { of gels }\end{array}$ & SD & $\begin{array}{l}\text { Density } \\
\text { of sols }\end{array}$ & SD & $\begin{array}{l}\text { Density } \\
\text { of gels }\end{array}$ & SD \\
\hline$\overline{F_{1}}$ & $2.405 .10^{-4}$ & $1.7 .10^{-7}$ & $1.0 .10^{-6}$ & 0 & 1.33850 & $4.9 .10^{-5}$ & 1.33875 & $5.2 .10^{-5}$ & 1.0284 & $5.10^{-4}$ & 1.0310 & $4.10^{-4}$ \\
\hline $\mathrm{F}_{1}^{\prime}$ & $2.425 .10^{-4}$ & $1.7 .10^{-7}$ & $1.5 .10^{-6}$ & 0 & 1.33900 & $4.7 .10^{-5}$ & 1.33875 & $4.2 .10^{-5}$ & 1.0299 & $4.10^{-4}$ & 1.0326 & $5.10^{-4}$ \\
\hline $\mathrm{F}_{2}$ & $2.435 .10^{-4}$ & $3.3 .10^{-7}$ & $5.10^{-7}$ & 0 & 1.33850 & $4.5 .10^{-5}$ & 1.33875 & $4.4 .10^{-5}$ & 1.0409 & $4.10^{-4}$ & 1.0423 & $4.10^{-4}$ \\
\hline $\mathrm{F}_{2}{ }_{2}$ & $2.445 .10^{-4}$ & $3.3 .10^{-7}$ & $5.10^{-7}$ & 0 & 1.33875 & $3.9 .10^{-5}$ & 1.33850 & $4.1 .10^{-5}$ & 1.0415 & $5.10^{-4}$ & 1.0439 & $4.10^{-4}$ \\
\hline $\mathrm{F}_{3}$ & $2.410 .10^{-4}$ & $1.7 .10^{-7}$ & $1.0 .10^{-6}$ & 0 & 1.33875 & $4.3 .10^{-5}$ & 1.33850 & $3.8 .10^{-5}$ & 1.0295 & $3.10^{-4}$ & 1.0318 & $3.10^{-4}$ \\
\hline $\mathrm{F}_{3}$ & $2.430 .10^{-4}$ & $1.7 .10^{-7}$ & $1.5 .10^{-6}$ & 0 & 1.33875 & $4.6 .10^{-5}$ & 1.33900 & $4.3 .10^{-5}$ & 1.0307 & $5.10^{-4}$ & 1.0337 & $4.10^{-4}$ \\
\hline
\end{tabular}

Table 2. Results of determination Conductivity, Refractive index and Density of sols and hydrogels.

\section{Results and Discussion}

\subsection{Preparation of Hydrogels}

The hydrogels formulation would take place thanks to the interactions of $\mathrm{N}$-acetyl-D-glucosamine, D-glucosamine, hydroxyl, amine, and bicarbonate ion groups which are present in the mixtures. Keeping the solutions cool allowed us to reduce the mixture entropy, to keep the linearity and flexibility of chitosan in solution and to prevent the liberation and evaporation of carbon dioxide. Keeping the linearity and flexibility of chitosan in solution would increase the contact surface of its chains allowing them to interact with themselves and with the maximum of surrounding available molecules in the mixtures, when the temperature increases [14-18]. In addition, at low temperature and at $\mathrm{pH}$ close to neutrality, a protective water layer around chitosan chains, prevents them interacting with themselves and with the surrounding available molecules in the mixtures. It is in this way, it also prevents gelling of the sol $[14,15,19]$. On the other hand, when the device warms up, there is a gradual transfer of heat from the hot body to the cold body and evolution towards a homogeneous temperature. This is the second principle of thermodynamics [20]. In this study, this effect is responsible of water elimination and $\mathrm{CO}_{2}$ liberation and evaporation. It also responsible of the decrease of density apparent charges of ammonium ions, the increase of mixtures entropy with dehydration causing destruction of the protective water layer. This allows chitosan chains to bind themselves and with surrounding available molecules via the interactions of coulombs, hydrophobes, Van der Waals and the hydrogen bonds which activate in mixtures with a dominant hydrophobic interactions effect. The effect resulting from these interactions leads to the hydrogels formation (Figure 1) [14-17, 21].

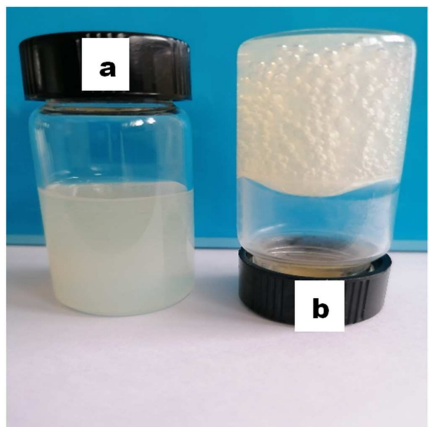

Figure 1. Sol (a)-gel (b) transition images. 


\subsection{Sol-gel Transition Time}

We will now focus on the variation of the sol-gel transition time as a function of the chitosan concentration, the final solution's $\mathrm{pH}$ and the ammonium ions concentration of each batch. The ammonium ions concentration is more important in the batch 2 . Regarding to the chitosan concentration, we noticed that for batch 1, the sol-gel transition time was equal to three minutes for $\mathrm{F}_{1}, \mathrm{~F}_{2}$, and $\mathrm{F}_{3}$ at $2.45 \%$ and was equal to two minutes at $2.55 \%$. The same results were noticed for the batch 2 , which included $\mathrm{F}_{1}, \mathrm{~F}_{2}$, and $\mathrm{F}_{3}$. The transition time from three to two minutes may be due to the decrease of the free energy at the interface between the chitosan chains and the surrounding available molecules. The decrease of the free energy is due to not only a decrease in the mobility and flexibility of the biopolymer (chitosan) but also a loss of entropy effects compensated by enthalpy effects, the mobility of counter ions, surrounding molecules and elimination of water molecules initially present in the sol. Indeed, the enthalpic effect increase when the biopolymer (chitosan) concentration increase [22, 23]. On the other hand, these results are comparable to those realized by Jingjing $\mathrm{Li}$ and $a l$ which showed that time transition sol-gel decrease when the biopolymer concentration increase [24]. Relative to the final solution's $\mathrm{pH}$ and the ammonium ions concentration, we noticed that the sol-gel transition time was equal to three minutes at pH6.8 and pH6.85 and for batches 1 and 2 at $2.45 \%$ of chitosan. In addition, the sol-gel transition time was equal to two minutes at $\mathrm{pH} 6.8$ and $\mathrm{pH} 6.85$ for the same batches at $2.55 \%$ of chitosan. In short, the sol-gel transition time did not vary as function of the final solution's $\mathrm{pH}$ and the ammonium ions concentration. These results are comparable to those which were described by Burgess and Singh in 1993 who had rapported that the sensibility of complex formation was significant if the variation of $\mathrm{pH}$ reached 0,5 units [25]. With regard to ammonium concentration, these results may be attributed to the fact that the difference of apparent charge density of ammonium ion concentration between the two batches was too small that additional electrostatic interactions did not take place [26] or gelation phenomenon would take place in secondes order. Indeed, in batch 2, eudragitE100 was used at low concentration $(0.0245$ and $0.0255 \%)$. For all formulations, the sol-gel transition time is more longer than the passage time of a liquid food from the mouth to the newborns stomach (2-3 mn Vs 4-5s) [27, 28]. The half gastric emptying time is also longer than the sol-gel transition time (180-240 mn Vs 2-3mn) [29]. For this purpose, the gelation will take place in his stomach.

\subsection{Physicochemical Characterization of Formulations}

\subsubsection{Visual and Organoleptic Aspect}

The sol and hydrogels had almost the same aspect because their densities and refractive index are comparable (Table 2) despite the presence of bubbles in the hydrogels which reflects the liberation and evaporation of $\mathrm{CO}_{2}$. In addition, the sol and hydrogels had a homogeneous appearance on the visual aspect. They were also odorless. This latter result allow to newborns to have a better acceptability for hydrogels and improves their treatment compliance.

\subsubsection{Conductivity, Refractive Index and Density}

The conductivities values of hydrogels were weaker than those measured with the sol. The decrease of conductivities in the hydrogels can be explained by the fact that the elimination of water molecules, the liberation and evaporation of $\mathrm{CO}_{2}$ and the presence of networks during the formation of hydrogels, leads to a decrease of ions charges. Besides, this decrease is more pronounced when the biopolymer (chitosan) concentration increases in the formulations (Table 2) [17]. This confirms the results of the sol-gel transition time discussed above because it decreases when conductivity decreases in the gel. Regarding to the chitosan concentration and at final solution's pH6.85, we noticed for the batch 1 that, the conductivity value was equal to $1.0 .10^{-6} \mathrm{~S} / \mathrm{cm}$ for $\mathrm{F}_{1}$ at $2.45 \%$ and was equal to $5.10^{-7} \mathrm{~S} / \mathrm{cm}$ for $\mathrm{F}_{2}$ at $2.55 \%$. For the batch 2 , noticed that, the conductivity value was equal to $1.510^{-6} \mathrm{~S} / \mathrm{cm}$ for $\mathrm{F}_{1}{ }_{1}$ at $2.45 \%$ and was equal to $5.10^{-7} \mathrm{~S} / \mathrm{cm}$ for $\mathrm{F}_{2}{ }_{2}$ at $2.55 \%$. These results can allows us to say that repulsion interactions would be more important in the formulations included the batch 2 than those included the bath 1 at $2.45 \%$ and decrease when chitosan concentration increased. Moreover, chitosan concentration had greatly influence the conductivity of hydrogels while the presence of eudragitE100's influence is weaker at $2.45 \%$. For the final solution's $\mathrm{pH}$, the conductivity of the sol increased when the $\mathrm{pH}$ decreased while it did not vary in the hydrogels (Table 2). These results confirm also the study rapported by Burgess and Singh in 1993. In addition, conductivity of hydrogels between the formulations of bath 1 and 2 varied from 0 to $5.10^{-7} \mathrm{~S} / \mathrm{cm}$ (Table 2). These results confirm the fact that time transition of hydrogels from the batch 1 and 2 did not vary because this conductivity value is too small. Therefore, additionnal electrostatic interaction did not take place between the formulations of the two batches. On the other hand, we noticed that the refractive index and densities of sol and hydrogels are comparable (Table 2). These results justify the resemblance of the aspects between the sol and the hydrogels. Our Conductivity and refractive index values are comparable to the study rapported by Baghera and al. in 2019 and Malinkinad and al. in 2016 respectively [30, 31].

\subsubsection{Scanning Electron Microscopy}

Microscopy allowed us to visualize the hydrogels morphology which is presented in figure 3 .

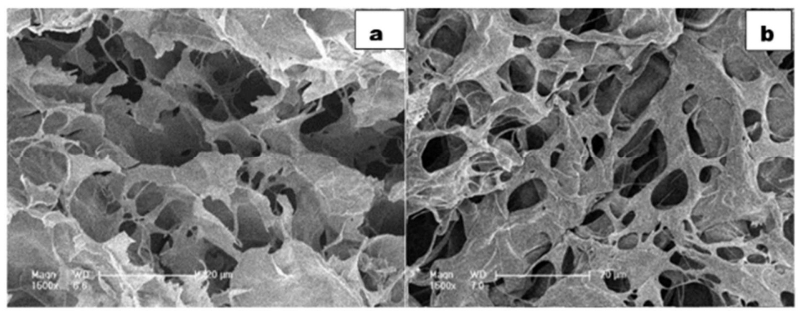

Figure 2. SEM images showing morphology of 2.45 (a) and $2.55 \%$ (b) of chitosan-based hydrogels with or without eudragit E100. 
These results showed us that the formulated hydrogels had pores whose size differs depending on the formulation. This result can be explained by the different interactions that are responsible of hydrogels formation. Therefore, the bonding energy in any space of hydrogels is not the same. This will lead to a system whose internal structure will be heterogeneous. Moreover, polysaccharide hydrogels have generally a semi-crystalline or amorphous structure $[32,33]$.

\subsubsection{In vitro Release Study and Mathematical Fitting of the Phenobarbital Release Profiles}

The percentage of phenobarbital released as a function of time is presented in figures 3 to 10 . These figures show the influence of the presence of eudragitE100, the chitosan concentration, and the final solution's $\mathrm{pH}$ on the phenobarbital release from hydrogels. The latter were placed in dissolution medium $\mathrm{pH}$ equal to 5 simulating the small intestinal liquids of newborns (prematures and term newborns), at $\mathrm{pH}$ equal to 7 for the gastric liquids of term newborns and at $\mathrm{pH}$ equal to 8 for the gastric liquids of prematures $[8,10]$.

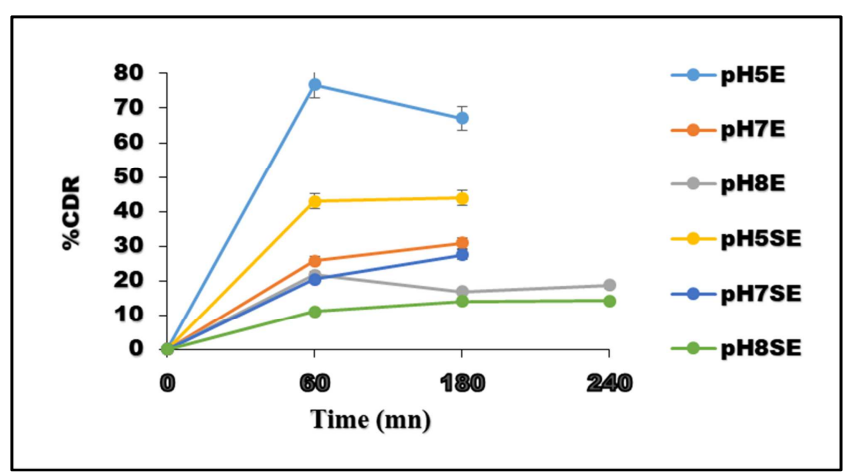

Figure 3. Pourcentages of cumulative drug release (\%CDR) as function of time for $F_{1}$ and $F^{\prime}{ }_{1}$. pH5E, pH7E and pH8E mean dissolution medium 's $p H$ equal to 5,7 and 8 for hydrogels containing eudragitE100. pH5SE, pH7SE and pH8SE mean dissolution medium's $p H$ equal to 5,7 and 8 for hydrogels without eudragitE100.

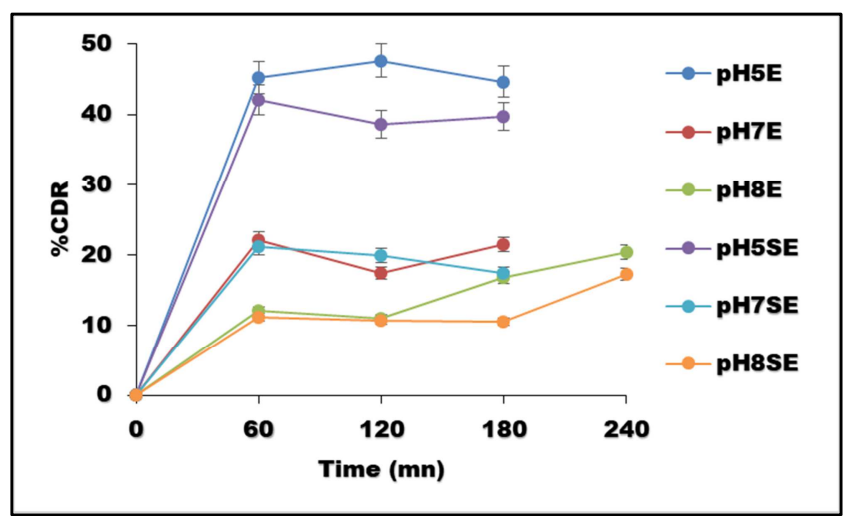

Figure 4. Pourcentages of cumulative drug release (\%CDR) as function of time for $F_{2}$ and $F_{2}{ }_{2} . p H 5 E, p H 7 E$ and $p H 8 E$ mean dissolution medium's $p H$ equal to 5,7 and 8 for hydrogels containing eudragitE100. pH5SE, pH7SE and pH8SE mean dissolution medium's $p H$ equal to 5,7 and 8 for hydrogels without eudragitE100.

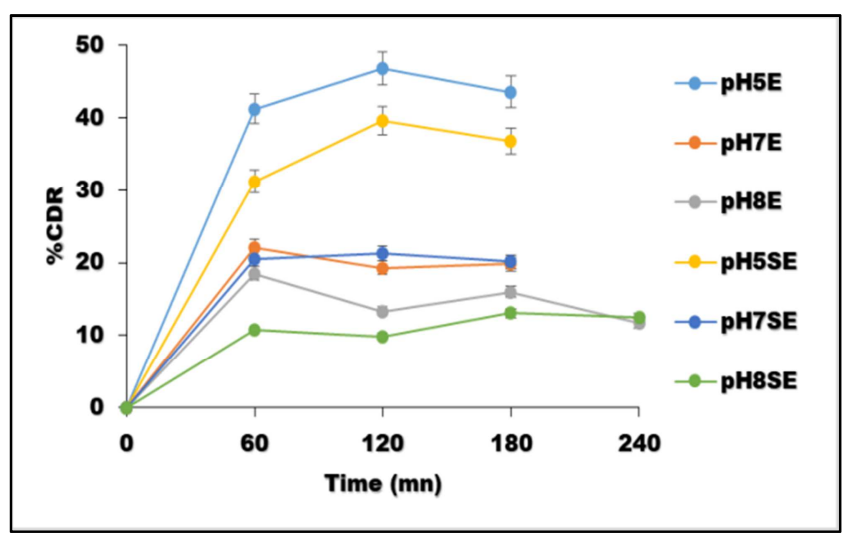

Figure 5. Pourcentages of cumulative drug release (\%CDR) as function of time for $F_{3}$ and $F_{3}{ }_{3}$. pH5E, pH7E and pH8E mean dissolution medium's $p H$ equal to 5,7 and 8 for hydrogels containing eudragitE100. pH5SE, pH7SE and $p H 8 S E$ mean dissolution medium's $p H$ equal to 5,7 and 8 for hydrogels without eudragitE100.

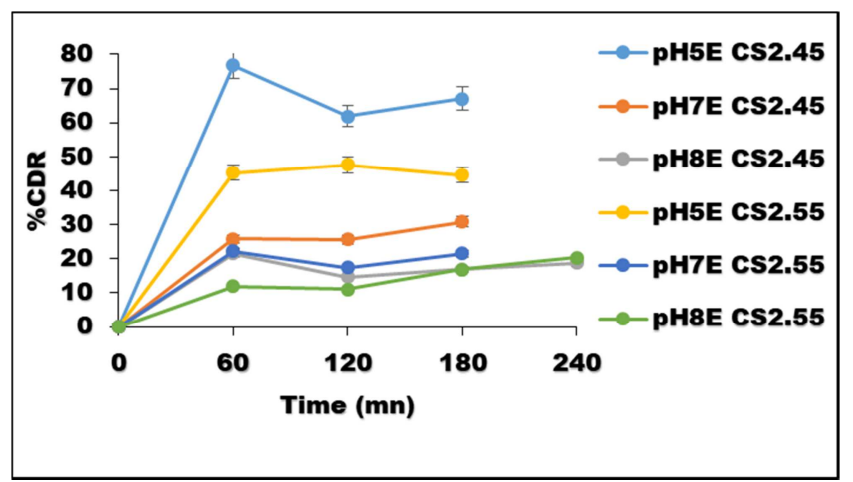

Figure 6. Pourcentages of cumulative drug release (\%CDR) as function of time for $F_{1}^{\prime}$ and $F_{2}{ }_{2}$. pH5ECS2.45, pH7ECS2.45 and pH8ECS2.45 mean dissolution medium's $p H$ equal to 5,7 and 8 for hydrogels containing $2.45 \%$ of chitosan and eudragitE100. pH5ECS2.55, pH7ECS2.55 and pH8ECS2.55 mean dissolution medium's $p H$ equal to 5,7 and 8 for hydrogels containing $2.55 \%$ of chitosan and eudragitE 100 .

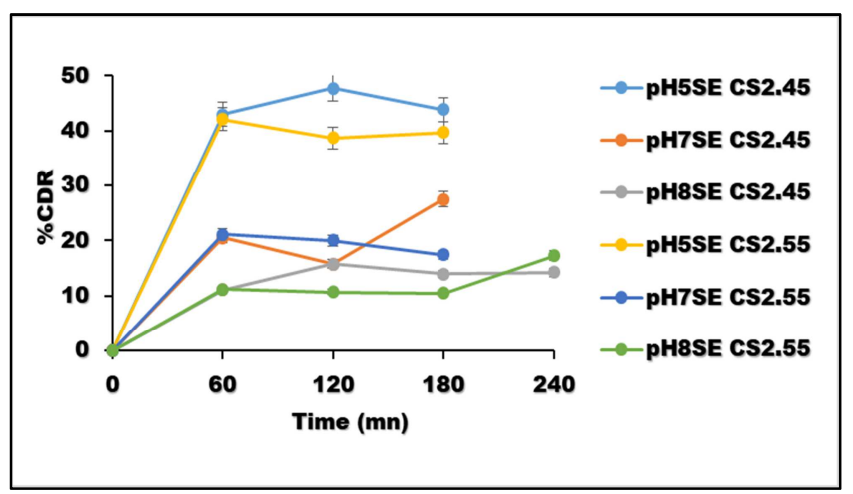

Figure 7. Pourcentages of cumulative drug release (\%CDR) as function of time for $F_{1}$ and $F_{2}$. pH5ECS2.45, pH7ECS2.45 and pH8ECS2.45 mean dissolution medium's pH equal to 5, 7 and 8 for hydrogels containing $2.45 \%$ of chitosan and without eudragitE100. pH5ECS2.55, pH7ECS2.55 and pH8ECS2.55 mean dissolution medium's $\mathrm{pH}$ equal to 5, 7 and 8 for hydrogels containing $2.55 \%$ of chitosan and without eudragitE100. 


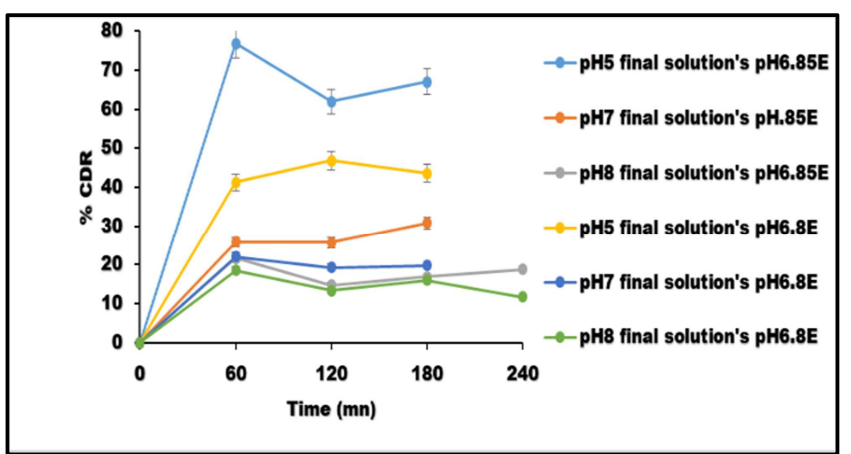

Figure 8. Pourcentages of cumulative drug release (\%CDR) as function of time for $F_{1}{ }_{1}$ and $F_{3}{ }_{3}$. pH5 final solution's pH6.85E, $p H 7$ final solution's pH6.85E and pH8 final solution's pH6.85E mean dissolution medium's $p H$ equal to 5, 7 and 8 for the $\mathrm{pH}$ sol equal to 6.85 and eudragitE 100. pH5 final solution's $\mathrm{pH6.8E}$, $\mathrm{pH} 7$ final solution's $\mathrm{pH} 6.8 \mathrm{E}$ and $\mathrm{pH} 8$ final solution's pH6.8E mean dissolution medium's $\mathrm{pH}$ equal to 5,7 and 8 for the $\mathrm{pH}$ sol equal to 6.8 and eudragitE100.

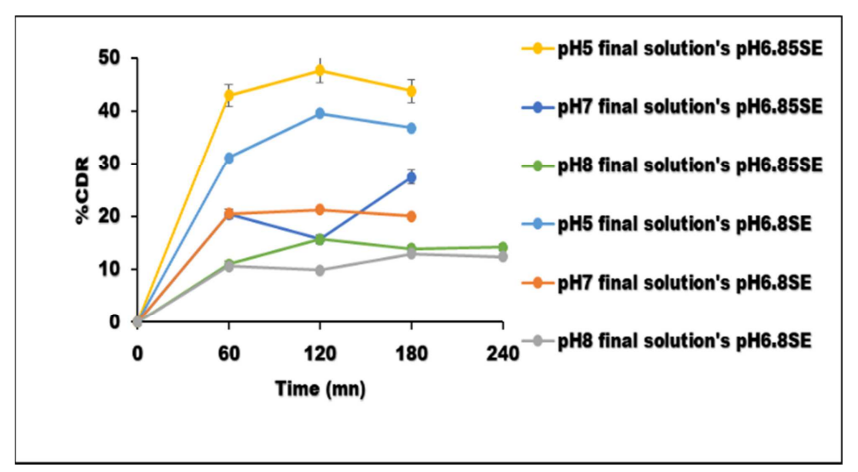

Figure 9. Pourcentages of cumulative drug release (\%CDR) as function of time for $F_{1}$ and $F_{3}$. pH5 final solution's pH6.85SE, pH7 final solution's pH6.85SE and pH8 final solution's pH6.85SE mean dissolution medium's $p H$ equal to 5, 7 and 8 for the $\mathrm{pH}$ sol equal to 6.85 and without eudragitE100. pH5 final solution's $p H 6.8 S E$, $p H 7$ final solution's $p H 6.8 S E$ and $p H 8$ final solution's $p H 6.8 S E$ mean dissolution medium's $p H$ equal to 5, 7 and 8 for the pH sol equal to 6.8 and without eudragitE100.

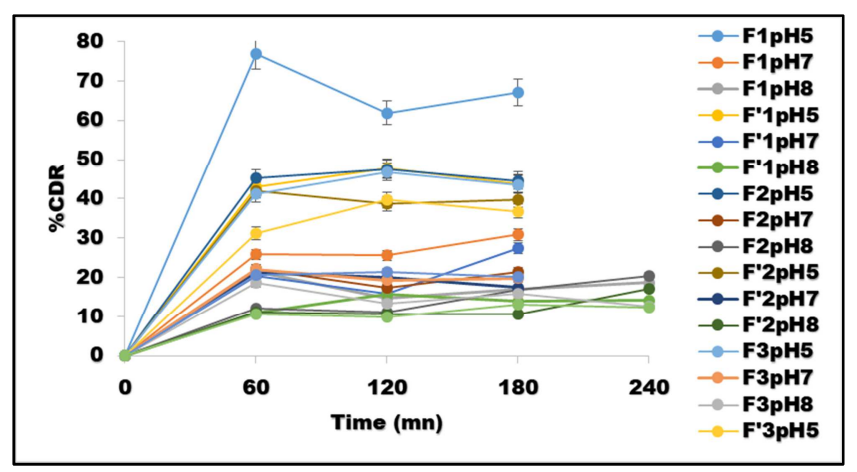

Figure 10. Pourcentages of cumulative drug release $(\% C D R)$ as function of time for all the hydrogels. $F_{1}, F_{2}, F_{3}$ and $F_{1}^{\prime}, F_{2}{ }_{2}$ and $F_{3}^{\prime}$ represent the hydrogels without and with eudragitE100. $\mathrm{pH} 5 \mathrm{pH}$ pH8 represent the dissolution medium's $p H$ equal to 5,7 and 8 .

At $\mathrm{pH}$ equal to 5 , the presence of eudragitE100 and the variation of chitosan concentration in the hydrogels, influenced the phenobarbital release. Indeed, the phenobarbital release increases with the presence of eudragitE100 and decreases when the chitosan concentration increases. Besides, it was more pronounced at this $\mathrm{pH}$ for all hydrogels. Consequently, the highest cumulative percentages of phenobarbital released were also observed at this $\mathrm{pH}$ (around 30-70\%) for $180 \mathrm{mn}$ (Figures 3 to 7). These results had been confirmed above by the conductivities values [34]. These results can be attributed to the surplus of the amine groups present in chitosan and eudragit E100 which protonate more in an acid medium than in a neutral or basic medium [34]. The protonation of the amine groups leads to the electrostatic repulsion of their chains, to the solubilization of the amine and hydroxyl groups, which creates a space favoring an entry of the water molecules into the hydrogels [35]. The entry of water molecules causes the hydration of the chitosan chains and gradually decreases the interactions of coulomb, Van der Waals, hydrophobes and increases the electro-osmotic pressure initially present in the hydrogels. At the same time, it promotes the contractile force of hydrogels networks which depends on their elasticity and which is inversely proportional to the biopolymer (chitosan) concentration [35-39]. Still at $\mathrm{pH}$ equal to 5, the variation final solution's $\mathrm{pH}$ influenced also the phenobarbital release. Indeed, for the same chitosan concentration, we noticed that the final solution with a higher $\mathrm{pH}$, released more phenobarbital (Figures 8 and 9). This result can be explained by the fact that the $\mathrm{pH}$ of gels is proportional to the porosity and inversely proportional to the mechanical strengths of hydrogels [40]. At $\mathrm{pH}$ equal to 7 and $\mathrm{pH}$ equal to 8 , the influence of the parameters mentioned above is weak. In these dissolution medium, we noted that the phenobarbital release is less pronounced with lower cumulative percentages of phenobarbital released, especially at $\mathrm{pH}$ equal to 7 (around $20-30 \%$ ) and at $\mathrm{pH}$ equal to 8 (around 10-20\%) (Figures 3 to 9). These results can be attributed to the fact that in basic or neutral medium, the protonation of the amine groups is weak, because the majority counter-ions in the dissolution medium can interact with the charged sites of the biopolymer by reducing the electrostatic repulsion and the hydrogels elasticity [36, 37, 39]. The result of all these effects allowed to release a more important phenobarbital amount in the medium simulating the intestinal liquids ( $\mathrm{pH}$ equal to 5) than in those simulating the stomach of the newborns ( $\mathrm{pH}$ equal to 7 and 8). In addition, at $\mathrm{pH}$ equal to 5, we also observed a minimum phenobarbital cumulative percentages released for $\mathrm{F}_{3}$ with values around $30 \%$ for $180 \mathrm{mn}$ (Figure 10). At $\mathrm{pH}$ equal to 7 and $\mathrm{pH}$ equal to 8 , we observed cumulative percentages around 20 and $10 \%$ respectively for 180 and 240 $\mathrm{mn}$ (Figure 10). These results can also be explained by the fact that the $\mathrm{pH}$ of gels is proportional to the porosity and inversely proportional to the mechanical strengths of the hydrogels. In addition, $\mathrm{F}_{3}$ having a smaller final solution's $\mathrm{pH}$ and devoid of eudragit E100, the size of its pores would therefore be smaller with high mechanical strength for hydrogels with the same chitosan concentration [40]. We also noticed that the phenobarbital release from hydrogels is time dependent and occurs in sequential form. It translate a pulsatile release profile $[39,41]$. The pulsatile release profiles can be explained by the fact that the hydration, dissolution and erosion of the biopolymers which active 
molecule conditions release take place gradually and go from site to site in biopolymers [35, 39, 41, 42]. And since in this study, the structure of hydrogels was heterogeneous, with this mechanism action of dissolution medium on the hydrogels, a pulsatile release mode was very possible. The KorsmeyerPeppas model was used to fit the phenobarbital release profiles from hydrogels. The equation used is as follows:

$$
\mathrm{Y}=\mathrm{K}_{\mathrm{kp}} \mathrm{t}^{\mathrm{n}} \text { with }
$$

$\mathrm{Y}=$ Cumulative fraction of phenobarbital released at time $\mathrm{t}$;

$\mathrm{K}_{\mathrm{kp}}=$ Korsmeyer-Peppas kinetic constant;

$\mathrm{n}=$ Diffusional exponent which indicates the phenobarbital release mechanism [43].

The parameters for fitting the phenobarbital release profiles are presented in table 3 . These results indicate that the phenobarbital release from hydrogels follows a water diffusion-controlled process because their $\mathrm{n}$ is less than 0.5 . Indeed, the relaxation of the chains during the swelling of the hydrogels contributes to the release, but the diffusion seems to be the major phenomenon in the release mechanism considering the fact that phenobarbital is a small molecule and in this study we used it at a very low concentration $(0.0293 \mathrm{mg} / \mathrm{ml})$ [43]. Therefore, the meshes of hydrogels could be larger than the size of phenobarbital. Among all the formulations, $\mathrm{F}_{2}, \mathrm{~F}_{2}$ and $\mathrm{F}_{3}{ }_{3}$ are suitable for this model for a potential application in the term newborns ( $\mathrm{pH}$ equal to $5, \mathrm{pH}$ equal to 7 ). On the other hand, $\mathrm{F}_{1}$ is suitable for a potential application in prematures ( $\mathrm{pH}$ equal to 5, $\mathrm{pH}$ equal to 8). In addition $\mathrm{F}_{1}{ }_{1}$ and $\mathrm{F}_{3}$ are suitable for prematures and term newborns $(\mathrm{pH}$ equal to $5, \mathrm{pH}$ equal to $7, \mathrm{pH}$ equal to 8 ). In short, the formulation $\mathrm{F}_{3}$ is the most suitable for this model according the $\mathrm{R}^{2}$ of the parameters for fitting the phenobarbital release profiles (Table 3 ) $[8,10$, $39,41,43-45]$.

Table 3. Parameters for fitting phenobarbital release profiles.

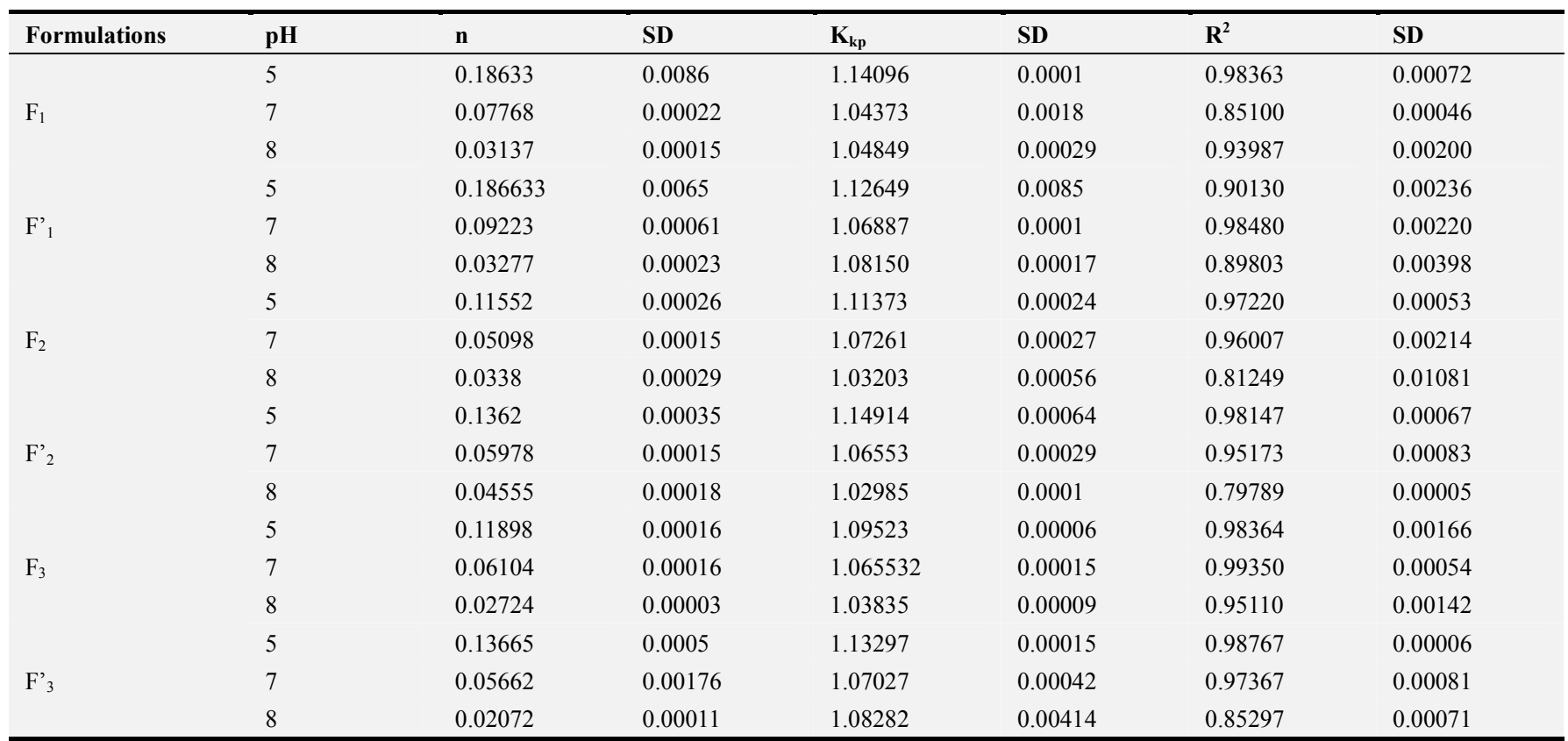

\section{Conclusion}

The Analysis of the phenobarbital release profiles in vitro and their fitting according to the Korsmeyer-Peppas equation showed that the different matrix used, lend themselves to a phenobarbital pulsatile release with a more important amount in the medium simulating the small intestine of the newborns than that simulating his stomach. This work can serve as a starting point for the design and development of oral chitosan-based hydrogels able to release phenobarbital in the small intestine of the newborns with suitable doses, an easily administered dosage form, improved bioavailability and efficiency with less side effects. The next step would be to study the viscosity, rheology, stability, cytotoxicity, and their dynamic structures.

\section{References}

[1] El-Dib, M. and J. S. Soul, The use of phenobarbital and other anti-seizure drugs in newborns. Seminars in Fetal and Neonatal Medicine, 2017. 22 (5): p. 321-327.

[2] Eker, H. E., et al. Children on phenobarbital monotherapy requires more sedatives during MRI. Pediatric Anesthesia, 2011. 21 (10): p. 998-1002.

[3] Guerrini, R., Epilepsy in children. The Lancet, 2006. 367 (9509): p. 499-524.

[4] Patsalos, P. N. and E. Perucca, Clinically important drug interactions in epilepsy: interactions between antiepileptic drugs and other drugs. The Lancet Neurology, 2003.2 (8): p. 473-481.

[5] Ferrendelli, J. A., Pharmacology of Antiepileptic Drugs. Epilepsia, 1987. 28 (s3): p. S14-S16. 
[6] Kaputu-Kalala-Malu, C., Epilepsies et crises épileptiques aiguës chez l'enfant en Afrique subsaharienne: défis et espoirs. Pan African Medical Journal, 2016. 23.

[7] Ivanovska, V., et al., Pediatric Drug Formulations: A Review of Challenges and Progress. Pediatrics, 2014. 134 (2): p. 361372.

[8] Batchelor, H. K. and J. F. Marriott, Paediatric pharmacokinetics: key considerations. Br J Clin Pharmacol, 2015. 79 (3): p. 395-404.

[9] Marsot, A., et al., Pharmacokinetics and absolute bioavailability of phenobarbital in neonates and young infants, a population pharmacokinetic modelling approach. Fundam Clin Pharmacol, 2014. 28 (4): p. 465-471.

[10] Neut, C., C. Romond, and H. Beerens, Contribution à l'étude de la répartition des espèces de Bifidobacterium dans la flore fécale de nourrissons alimentés soit au sein, soit par des laits maternisés. Reproduction Nutrition Développement, 1980. 20 (5B): p. 1679-1684.

[11] Hanning, S. M., et al., Patient centric formulations for paediatrics and geriatrics: Similarities and differences. Int J Pharm, 2016. 512 (2): p. 355-359.

[12] Zhang, A., et al., Recent advances in stimuli-responsive polymer systems for remotely controlled drug release. Progress in Polymer Science, 2019. 99: p. 101164.

[13] Guzmán, M. L., R. H. Manzo, and M. E. Olivera, Eudragit E100 as a Drug Carrier: The Remarkable Affinity of Phosphate Ester for Dimethylamine. Mol Pharm, 2012. 9 (9): p. 2424-2433.

[14] Philippova, O. E. and E. V. Korchagina, Chitosan and its hydrophobic derivatives: Preparation and aggregation in dilute aqueous solutions. Polymer Science Series A, 2012. 54 (7): p. $552-572$.

[15] Hassani, L. N., F. Hendra, and K. Bouchemal, Autoassociative amphiphilic polysaccharides as drug delivery systems. Drug Discov Today, 2012. 17 (11-12): p. 608-614.

[16] Liu, L., et al., Smart gelation of chitosan solution in the presence of $\mathrm{NaHCO} 3$ for injectable drug delivery system. Int J Pharm, 2011. 414 (1-2): p. 6-15.

[17] Cerchiara, T., et al. Physically cross-linked chitosan hydrogels as topical vehicles for hydrophilic drugs. Journal of Pharmacy and Pharmacology, 2002. 54 (11): p. 1453-1459.

[18] Aymard, P., et al. Influence of thermal history on the structural and mechanical properties of agarose gels. Biopolymers, 2001. 59 (3): p. 131-144.

[19] Srividya, B., R. M. Cardoza, and P. D. Amin, Sustained ophthalmic delivery of ofloxacin from a $\mathrm{pH}$ triggered in situ gelling system. Journal of Controlled Release, 2001. 73 (2-3): p. 205-211.

[20] Aberkane, L. 1., et al., Thermodynamic Characterization of Acacia Gum- $\beta$-Lactoglobulin Complex Coacervation. Langmuir, 2010. 26 (15): p. 12523-12533.

[21] Desbrières, J., C. Martinez, and M. Rinaudo, Hydrophobic derivatives of chitosan: Characterization and rheological behaviour. Int J Biol Macromol, 1996. 19 (1): p. 21-28.

[22] S. Damodaran et A. Paraf (Eds), Food Proteins and their applications, 1997; p.171-198. New York: Marcel Dekker, Inc.
[23] Piculell, L. et al. Factor Determining Phase Behaviour of Multi-Component Polymer Systems. S. E Harding, S. E. Hill et J. R. Mitchell (Eds). Biopolymer Mixtures, 1995; p.13-36, Nottingham: Notttingham University Press.

[24] Jingjing and al. In vitro and in vivo evalution of 3D biodegradable thermo/pH sensitive sol-gel reversible hydroxybutyl chitosan hydrogel. Materials science et Engineering C, china, 2020; 108:p.110419.

[25] Burgess, D. J. and Singh, O. N. Gupta, Spontaneous formation of small sized albumin/accacia coarcervate particules. J. Pharm. Pharmaco., 1993;43: p.232-236.

[26] Wen Y-p. and Dubin, P. L. Potentiometric Studies of the interaction of bovine serum albumin and Poly (dimethyldiallylammonium chloride. Macromolecules, 1997, 30:p.7856-7861.

[27] Kaye, J. L., Review of paediatric gastrointestinal physiology data relevant to oral drug delivery. International Journal of Clinical Pharmacy, 2011. 33 (1): p. 20-24.

[28] Bowles, A., et al. Specific aspects of gastro-intestinal transit in children for drug delivery design. Int J Pharm, 2010. 395 (1-2): p. 37-43.

[29] Strolin Benedetti, M., R. Whomsley, and E. L. Baltes, Differences in absorption, distribution, metabolism and excretion of xenobiotics between the paediatric and adult populations. Expert Opin Drug Metab Toxicol, 2005. 1 (3): p. 447-471.

[30] Bagher, Z., et al., Conductive hydrogel based on chitosananiline pentamer/gelatin/agarose significantly promoted motor neuron-like cells differentiation of human olfactory ectomesenchymal stem cells. Materials Science and Engineering: C, 2019. 101: p. 243-253.

[31] Malinkina, O. N., A. M. Sobolev, and A. B. Shipovskaya, Hybrid Nanogels Based on Chitosan Hydrochloride-Ascorbate Derived by Sol-Gel Biomimetic Synthesis. BioNanoScience, 2016. 6 (2): p. 157-161.

[32] Nie, J., et al. Construction of ordered structure in polysaccharide hydrogel: A review. Carbohydr Polym, 2019. 205: p. 225-235.

[33] Ma, S., et al., Structural hydrogels. Polymer (Guildf), 2016. 98: p. 516-535.

[34] Vasheghani-Farahani, E., et al., Swelling of ionic gels in electrolyte solutions. Ind Eng Chem Res, 1990. 29 (4): p. 554560 .

[35] Tcharkhtchi, A., P. Y. Bronnec, and J. Verdu, Water absorption characteristics of diglycidylether of butane diol-3, 5-diethyl-2, 4-diaminotoluene networks. Polymer (Guildf), 2000. 41 (15): p. $5777-5785$.

[36] Ata, S., et al., Loading of Cefixime to $\mathrm{pH}$ sensitive chitosan based hydrogel and investigation of controlled release kinetics. Int J Biol Macromol, 2019.

[37] Wang, Y., et al., Chitosan cross-linked poly (acrylic acid) hydrogels: Drug release control and mechanism. Colloids and Surfaces B: Biointerfaces, 2017. 152: p. 252-259.

[38] Gupta, P., K. Vermani, and S. Garg, Hydrogels: from controlled release to $\mathrm{pH}$-responsive drug delivery. Drug Discov Today, 2002.7 (10): p. 569-579. 
[39] Peppas, N., Hydrogels in pharmaceutical formulations. European Journal of Pharmaceutics and Biopharmaceutics, 2000. 50 (1): p. 27-46.

[40] Qu, X., A. Wirsén, and A. C. Albertsson, Novel pH-sensitive chitosan hydrogels: swelling behavior and states of water. Polymer (Guildf), 2000. 41 (12): p. 4589-4598.

[41] Gazzaniga, A., et al., Oral pulsatile delivery systems based on swellable hydrophilic polymers. European Journal of Pharmaceutics and Biopharmaceutics, 2008. 68 (1): p. 11-18.

[42] Maroni, A., et al., Erodible drug delivery systems for timecontrolled release into the gastrointestinal tract. Journal of Drug Delivery Science and Technology, 2016. 32: p. 229-235.
[43] Bacaita, E. S., et al., Phases in the temporal multiscale evolution of the drug release mechanism in IPN-type chitosan based hydrogels. Phys. Chem. Chem. Phys., 2014. 16 (47): p. 25896-25905.

[44] Jain, D., et al., Recent technologies in pulsatile drug delivery systems. Biomatter, 2014. 1 (1): p. 57-65.

[45] Maroni, A., et al., Oral pulsatile delivery: Rationale and chronopharmaceutical formulations. Int J Pharm, 2010. 398 (1-2): p. 1-8. 